Chapter 4

\title{
Regenerative Medicine in the Oral and Maxillofacial Region
}

\author{
Hideto Saijo \\ Additional information is available at the end of the chapter \\ http://dx.doi.org/10.5772/58903
}

\section{Introduction}

The bone reconstruction has conventionally used autologous bone, allograft bone, or artificial bone in the maxillofacial resigns. Since bone reconstruction using artificial bone avoids the highly invasive harvesting of bone needed for autologous bone grafts, many different types of artificial bone have been developed. The requirements of artificial bone are that it (1) has good surgical manipulability, (2) can conform well to the defective area, (3) has moderate strength, and (4) has the capacity to induce bone regeneration. Artificial bone is marketed worldwide as blocks, in particulate form, or as paste, but no artificial bone has yet been developed that satisfies all these requirements. The ability to reproduce shapes is particularly important, and from the point of view of esthetics, it is regarded as the key to success in the field of cranio-maxillo-facial reconstruction. Reconstruction using custom-made artificial bone and titanium mesh trays has been reported, (Iino $\mathrm{M}$ et al., 2009). but since the reconstructions were made using computer-aided design, it would be difficult to customize the reconstruction to the level of detail that surgical operators expect. The clinical application of custom-made artificial bone that we have developed is described.

\section{Materials and methods}

The subjects were patients with a defect of the maxilla or mandible that was either congenital or acquired through trauma. Cranial plain computed tomography (CT) imaging was carried out according to the usual acquisition protocols using the Aquilion 16 scanner (Toshiba Medical Systems Corporation, Japan) at $120 \mathrm{kV}, 300 \mathrm{~mA}, 0.5-\mathrm{mm}$ slice interval, and helical pitch of 16. CT images were saved in Digital Imaging and Communications in Medicine (DICOM) format for transmission over a network. CT values were determined for extracting 
bony tissue from the slice images, and the acquired 3-dimensional data were output as Standard Template Library (STL) files. The 3-dimensional form of the skeletal structure to be fabricated was designed using 3-D computer-aided design (CAD) on the basis of the STL data, and a solid model was fabricated using the Z406 inkjet powder layering device.

\subsection{Surgical simulations and artificial bone fabrication}

The surgical simulations were carried out by sculpting an original wax into defective areas or areas needing supplements of artificial bone on the solid models (Fig. 1). Since the response of soft tissues to changes differs depending on the individual, the wax needed to be shaped with the condition of the soft tissues taken into account. The simulation solid models were scanned by CT, and the CT data were output in DICOM format. Using the CAD data, the regions of artificial bone sculpted in wax were extracted (Fig. 2). Using the CAD system, the inner surface of the artificial bone structure that makes contact with the host bone and the holes for fixing the artificial bone in place were designed onto the extracted artificial bone data. The artificial bone was then fabricated using the Z4063D printer (DICO), an inkjet powder layering device. The material for fabricating the artificial bone was $\alpha$-tricalcium phosphate powder, with aqueous solution of polysaccharides as a sclerosing solution. The artificial bone thus formed was a calcium phosphate. (Saijo H et al., 2011).
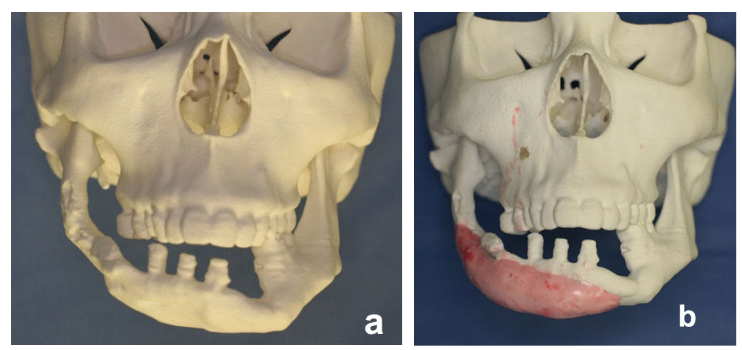

Figure 1. Simulation with wax. a: The three-dimensional model that was molded with gypsum. b: Simulation with the wax.
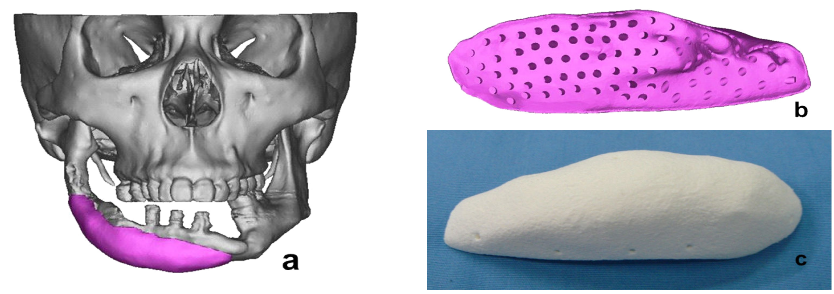

Figure 2. The design of artificial bone. a: The artificial bone designed by CAD. b: The inside view of the artificial bone in the CAD. The inside structure of the artificial bone can be freely simulated by CAD. c: The artificial bone that was made. 


\subsection{Surgical procedures and follow-up}

The operation was performed under general anesthetic, and the region was approached both from the mouth and externally. The periosteum was detached with care not to damage it, and the bone of the graft site was revealed. A trial insertion was made of the custom-made artificial bone, and changes in the soft tissues were checked. Where fixation was then carried out, holes for fixing were made using a surgical drill, and the artificial bone was fixed in place with absorbable polyglycolic suture in several places. The surgical incision was closed by covering the artificial bone with periosteum and making periosteal sutures in order to prevent any deviation resulting from movement of the artificial bone after surgery(Saijo $\mathrm{H}$ et al., 2008).

\section{Results}

Conventional autologous grafts take several hours because the autologous bone must be harvested and shaped, but the present procedure greatly reduced the operation time. During surgery, there was excellent conformity between the artificial bone and the host bone, and firm fixing was not needed. Even where comparatively large artificial bone was grafted and fixing was necessary, no problems were encountered with fixing in several places. Furthermore, at around 3 months after surgery, there were appearances of partial bone union between the artificial bones and the host bone tissue in some patients. In addition, the patients showed a high satisfaction with the facial features following surgery.

\subsection{Case 1 (Fig.3)}

The patient was 55 years old woman. The patient visited the clinic with a main complaint of facial asymmetry. She was treated for cancer on the left side of her tongue approximately 20 years previously with partial resection of the tongue and segmental resection of the mandible. The region was reconstructed with grafts of a rib and a pectoralis major musculo-cutaneous flap. Radiation therapy was subsequently administered as after-treatment. A graft from the left ilium was carried out 5 years later due to resorption of the first graft. Implants were subsequently placed on the maxilla and the mandible, and dental implants were fitted, but noticeable facial asymmetry remained.

Mandibular reconstructive surgery consisting of an artificial bone graft was carried out under general anesthetic. The approach was made from outside the mouth, as there was a scar present on the neck from the previous operation. The bone was detached by subperiosteal dissection and the host bone graft site revealed, and a trial insertion of the custom-made artificial bone was made. Improvement of the facial features was confirmed, and the artificial bone was fixed to the host bone with 2-0 Vicryl suture. After placement of the graft, the artificial bone was covered with part of the periosteum and the fatty layer, and the surgical wound was closed in the usual way. Presently, at over 1 year operation, the patient is progressing and there is improvement of the facial features. No inflammatory reaction or other abnormalities have been found, and CT images show the position of the artificial bone to be stable with partial bone union. 

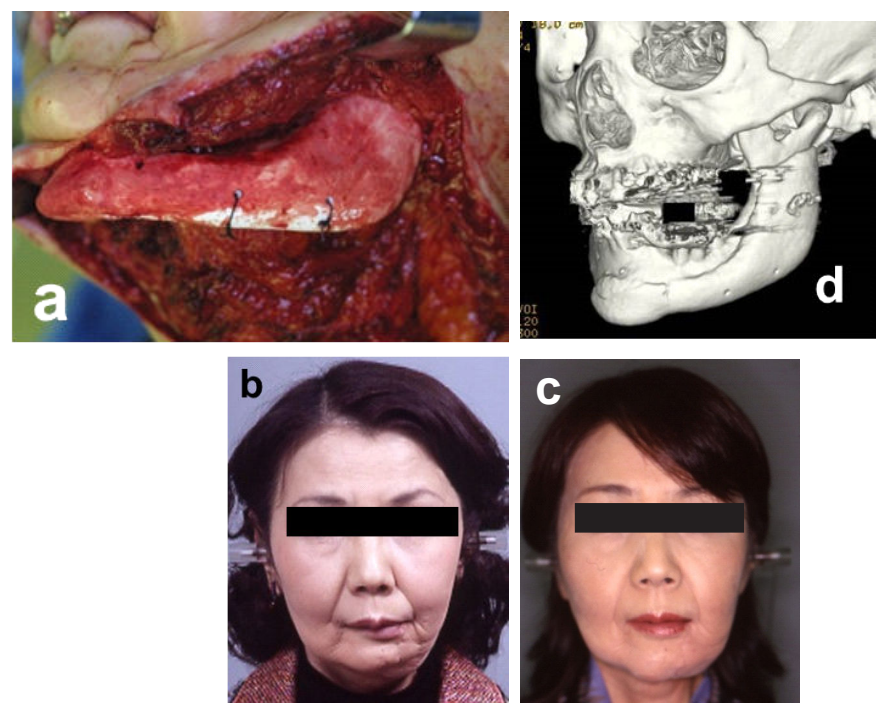

Figure 3. Case 1 a: During operation. b: Facial appearance of Patient 2 before surgery. c: Facial appearance at 2 year after surgery. $d$ : 3D CT image of the left lower jaw after surgery

\subsection{Case 2 (Fig.4)}

The patient was 31 years old woman. The patient was diagnosed with sarcoma of the mandible when she was 20 years old, and she was treated with segmental resection of the mandible and reconstruction. Mandibular reconstructive surgery was subsequently carried out on several occasions using donor bone from the rib and fibula, but she visited this department in 2001 due to bone resorption resulting from infection. Sequestrum removal and reconstruction using a vascularized fibular osteocutaneous flap were carried out at our department, but since there was still partial resorption due to infection, a graft of bone from a rib was carried out. The engrafted bone was subsequently stable, and dental implants were placed and the denture fitted. However, the facial features changed markedly as a result of the repeated infections and bone grafts, and an artificial bone graft was planned for the present treatment. The incision line from the previous surgery was used for the approach. The graft site bone was revealed, and a trial insertion of the custom-made artificial bone was made. Improvement of the facial features was confirmed, and the artificial bone was fixed to the host bone with several stitches of absorbable sutures. At 6 months after surgery, no abnormalities were found and there was improvement of the facial features. In addition, there were no abnormalities in the position of the artificial bone. 

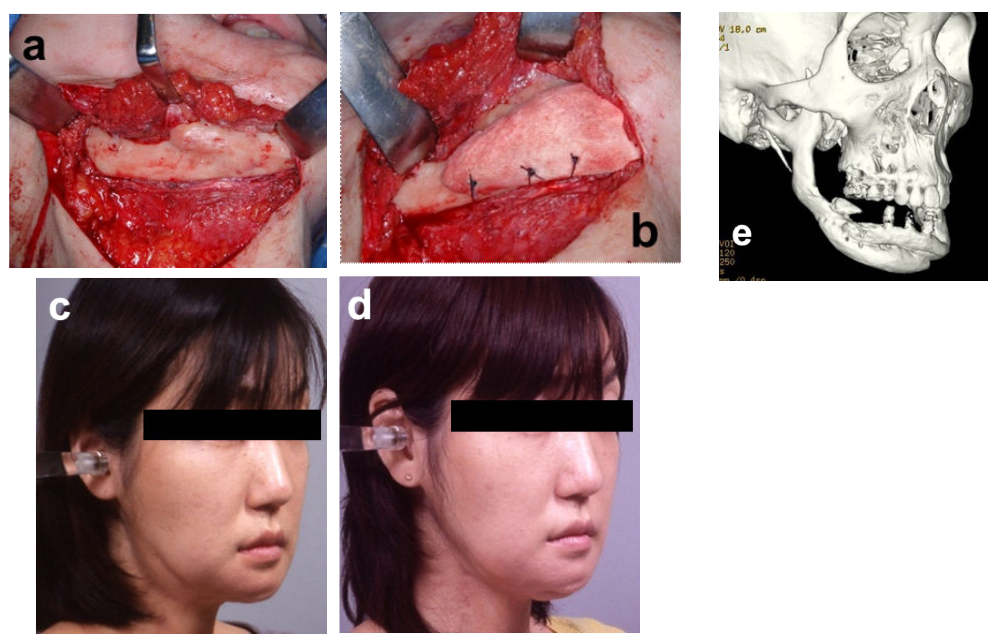

Figure 4. Case2 a, b: During operation c: Facial appearance before surgery d: Facial appearance at 1 year after surgery. e: 3D CT image of the left lower jaw after surgery

\section{Discussion}

Reconstruction with hard tissue is extremely useful in the treatment of bone defects in the cranio-maxillo-facial region. However, since reconstruction using autologous bone requires harvesting of the bone, massively invasive intervention is unavoidable. Particularly in cases of a large bone defect, it is not only necessary to use a revascularized osteocutaneous flap, which requires the microvessels to be anastomosed, but also craftsmanship is needed to shape the bone to conform to the graft site. Allograft bone carries the risk of infection, and there are also ethical issues, in addition to which the bone needs to be crafted into shape to conform to the graft site. The advantages of artificial bone are that no harvesting site is needed, it has excellent biocompatibility, and the surgical technique is brief and uncomplicated. The artificial bones currently being used around the world can be divided into hydroxyapatite15), $\alpha$-TCP ( $\alpha$-tricalcium phosphate), and $\alpha$-TCP ( $\alpha$-tricalcium phosphate). Each has its own characteristics, and the clinical application depends on the nature of the region of defective bone. Hydroxyapatite (HA) artificial bone has a strong affinity for biological tissue and is characterized by direct binding to bony tissue. This is because new bone growth is induced even inside pores by the invasion of collagen tissue, which is needed for ossification. In addition, HA has better mechanical strength than human bone, and it is widely used in clinical practice as a material for replenishing bone defects. (Saijo H et al., 2010). It has been made into commercially available products as porous blocks, granules, and paste. However, HA is highly crystalline and shows little resorption, almost all of it remaining in a stable condition. Thus, HA is processed at high temperature and is highly crystalline, and it is a biomaterial that is not readily resorbed. Tricalcium phosphate(TCP), on the other hand, is resorbed and replaced 
as the bone is restored. It has excellent tissue affinity and is osteoconductive. In other words, TCP is gradually converted to HA in vivo, and there is a mechanism whereby, as resorption of the artificial bone progresses, it is simultaneously replaced with new bone that forms as a result of invasion by osteoblasts. Thus, the artificial bone, which has excellent osteoconductivity and biocompatibility, is ultimately replaced by bone. In the present study, the main component of artificial bone was fine $\alpha$-TCP powder, which was made into a non-sintered hardened body of TCP through the use of a sclerosing solution. Such hardened bodies of TCP are likely to be biochemical precursors of HA, and not only do they have excellent biocompatibility like HA, but they are also artificial bones that are resorbed and replaced in vivo. It therefore appears likely that the artificial bones grafted in the present study will be replaced by bone.

Another feature of the present method is that the artificial bone is fabricated from a wax-up made by the surgical operator prior to surgery, using the 3-D cast. This means that the present method is able to reproduce the required shape more faithfully than conventional methods of fabrication. This results in better compatibility than existing types of custom-made artificial bone, so that practically no adjustments are necessary during the surgical procedure. Consequently, further reduction in the time needed for the surgical procedure can be expected. With the present patients, bone grafts were made to bone defects resulting from tumor or congenital anomalies. Where the graft was made to bone that had been grafted or lengthened, the surface of the bone presented an extremely complex form, and it is likely that there would have been limited compatibility with conventional methods. Furthermore, with the present method, the CAD artificial bone data can be readily used to fashion the inner surface of the artificial bone that makes contact with the host bone, allowing this region to be shaped freely. The present method allows the surgical operator to fabricate the custom-made artificial bone into the shape that patient's desires, and this aspect of the method is likely to make it extremely useful in clinical practice. The conformity of the artificial bone during the procedure was extremely good, so that there was no need for the complicated modifications to the shape that are necessary with conventional grafts of artificial bone. The raw material used with the present method is $\alpha-\mathrm{TCP}$, and the product is a non-sintered hydroxyapatite compact. Because its resorbability is better than that of artificial bone made from a sintered body, it is expected that the present artificial bone can be replaced by bone at an earlier stage. While the present artificial bone is not as strong as artificial bone made from a sintered body, it can withstand a force of $20 \mathrm{MPa}$, and this is unlikely to be a problem for use on non-load-bearing regions of facial bone. Furthermore, since artificial bone made from a sintered body is reported to shrink by around $15 \%$ during sintering, problems remain with regard to conformity and reproducibility. With the patients in the present study, union of the artificial bone and the host bone was found in some places on the CT images starting at around 3 months after surgery. This is probably because union of the present artificial bone with host bone is more rapid than with conventional types of artificial bone, since it is highly biocompatible and non-sintered. This is a huge advantage of non-sintered artificial bone. The most important aspect of artificial bone from the point of view of the surgical procedure of reconstruction is the fixing of the artificial bone. When calcium phosphate makes contact with the surface of bone, fibrous connective tissue appears at an early stage, causing union with the bone. However, any postoperative disturb- 
ance between the artificial bone and the host bone is likely to impair union. In the present study, where a relatively large piece of artificial bone that required fixing in place was grafted, this was sutured with absorbable suture. However, a method of fixation needs to be devised in the future.

In the present cases, reconstruction was successfully performed using custom-made artificial bone, with a high level of patient satisfaction. No serious postoperative side effects attributable to the artificial bone were found, so that the safety of the artificial bone was confirmed. However, with the present fabrication method, the postoperative reaction of the patient's soft tissues depends on the intuition of the surgical operator. As even better artificial bone is developed in the future, recovery of shape to even higher degrees of accuracy and earlier bone fusion may be expected. In other words, one may anticipate the development of custom-made artificial bone designed on a system allowing prediction of the shape of the soft tissues following the artificial bone graft, and biologically active custom-made artificial bone loaded with adhesion factors. (Saijo H et al., 2009)

\title{
5. Conclusion
}

These artificial bones were safe and achieved dimensional compatibility along with good biodegradability and osteoconductivity. We believe that this study has provided the basis for the use of the inkjet printing technology for maxillofacial reconstruction surgery

\section{Author details}

\author{
Hideto Saijo
}

Oral-Maxillofacial Surgery, Dentistry and Orthodontics The University of Tokyo Hospital, Japan

\section{References}

[1] Iino $\mathrm{M}$ et al.,(2009). Evaluation of 15 mandibular reconstructions with Dumbach Titan Mesh-System and particulate cancellous bone and marrow harvested from bilateral posterior ilia. Oral Surg Oral Med Oral Pathol Oral Radiol Endod 107: 1-8, 2009

[2] Saijo H et al.,(2011). A novel method for designing and fabricating custom-made artificial bones. Int J Oral Maxillofac Surg. vol40, pp.955-960.

[3] Saijo H et al.,(2008). Clinical Application of Artificial Bone in the Maxillofacial Region. J Artif Organs. ; vol 11,pp.171-176 
54 New Trends in Tissue Engineering and Regenerative Medicine - Official Book of the Japanese Society for Regenerative Medicine

[4] Saijo H et al.,(2010). A bone harvesting method involving a surgical template prepared using a three-dimensional model Asian. J. Oral.Maxillofac. Surg. vol22,pp. 20-22

[5] Saijo H et al.,(2009). Maxillofacial reconstruction using custom-made artificial bones fabricated by inkjet printing technology. J Artif Organs vol.12, pp.200-205 